\title{
A tomographic setting for quasi-distribution functions
}

\author{
V.I. Man'ko ${ }^{a}$, G. Marmo $^{b}$, A. Simonib ${ }^{b}$ E.C.G. Sudarshan ${ }^{c}$, \\ F. Ventriglia ${ }^{b}$ \\ ${ }^{a}$ P.N.Lebedev Physical Institute, Leninskii Prospect 53, Moscow 119991, Russia \\ (e-mail: manko@na.infn.it) \\ ${ }^{b}$ Dipartimento di Scienze Fisiche dell' Università "Federico II" e Sezione INFN di Napoli, \\ Complesso Universitario di Monte S. Angelo, via Cintia, 80126 Naples, Italy \\ (e-mail: marmo@na.infn.it, simoni@na.infn.it, ventriglia@na.infn.it) \\ ${ }^{c}$ Department of Physics, University of Texas, Austin, Texas 78712, USA \\ (e-mail: sudarshan@physics.utexas.edu)
}

May 5, 2018

\begin{abstract}
The method of constructing the tomographic probability distributions describing quantum states in parallel with density operators is presented. Known examples of Husimi-Kano quasi-distribution and photon number tomography are reconsidered in the new setting. New tomographic schemes based on coherent states and nonlinear coherent states of deformed oscillators, including $q$-oscillators, are suggested. The associated identity decompositions providing Gram-Schmidt operators are explicitly given.

Key words Quantum tomograms, Coherent state tomograms, Photon number tomograms, Husimi-Kano quasi-distribution, Sudarshan's diagonal coherent state representation, $f-$ and $q$-oscillators.

PACS: 03.65-w, 03.65. Wj
\end{abstract}

\section{Introduction}

There are several representations of quantum states providing the possibility to present equivalent, but different in their form, formulations of quantum mechanics [1. The representations of quantum states are based on different integral transforms of the density operator [2, 3] taken in the position representation. The density operator in the position representation is mapped by means of the integral transforms either to Wigner quasi-distribution function [4, or HusimiKano $K$-function [5, 6]. In this paper we have decided to keep up with the original notations of the pioneer papers on the subject. Quasi-distributions are 
usually referred to as phase space representations of quantum states. Another important phase space representation is related to the Sudarshan's diagonal coherent state representation [7, 8. Recently the tomographic representations of quantum states was suggested [9, 10, 11] using the Radon integral transform of their Wigner functions. The tomographic representation exhibits some specific property in comparison with the other phase space representations. The tomographic probability distributions (tomograms) associated with quantum states are standard positive probability distributions. The mathematical mechanism of constructing the tomographic probabilities in abstract Hilbert spaces was elucidated in 12 for the finite dimensional case. Also infinite dimensional Hilbert spaces were considered in the context of mapping vectors and density operators onto tomographic probabilities in 13 .

The details of those tomographic approach constructions inspire to study other possibilities to know better and create new schemes of description of quantum states by positive functions. First of all, the Husimi-Kano $K$-function interpreted as quasi-distribution in the phase space representation is a real nonnegative bounded function of two variables, $q$ and $p$. Strictly speaking, these variables cannot be interpreted as the actual position and momentum since the uncertainty relations prohibit the existence of a distribution in the phase space depending on variables which cannot be measured simultaneously. Nevertheless, being a real non-negative and normalized function on "phase space", it has a chance to be interpreted from the point of view of the tomographic approach. One of the aims of the present work is to extend our analysis of the mathematical mechanism of constructing tomographic sets to extra situations, by including into the construction properties of the coherent states [14] and properties of $K$-function and Sudarshan's diagonal coherent state representation [15] in the sense of their relation to distributions (i.e., generalized functions). We call this extension coherent state tomography. From this point of view the Husimi-Kano $K$-function will be interpreted as a tomogram of a quantum state.

The coherent states are closely connected with linear vibrations (linear harmonic oscillator). The deformed oscillators, e.g. $q-$ oscillators [16, 17] and $f-$ oscillators [18, 19] are related at a classical level with specific non-linear vibrations, so that non-linear coherent states were introduced [20, 21] to describe the corresponding states of a non-linear quantum oscillator, which yields in the linearity limit the standard coherent state counterpart. Another goal of this work is to develop the $f$-deformed version of the tomographic approach and in particular to present, for a specific choice of the non-linearity coded by the function $f=f_{q}$, the $q$-deformed coherent state tomography.

Also, by using $f$-deformed coherent states, we develop the $f$-deformed version of the photon number tomography [22, 23, 24] as well as we obtain the identity decomposition for the deformed tomographies. In fact, we are addressing the general problem of constructing a map from a Hilbert space (or projective Hilbert space) onto a family of probability distributions (tomograms).

As in the usual formulation of quantum mechanics there are several schemes like Schrödinger picture, Heisenberg picture, Dirac picture, even in the tomographic approach exist several different schemes, like symplectic tomography, 
photon number tomography, optical tomography and so on. Our last aim is to point out the common general mechanism of constructing all these tomographic schemes and in this way to extend the list of the tomographies by including the deformed ones.

The paper is organized as follows. In section 2 a short account of our construction of a tomographic setting in abstract Hilbert spaces is given. In section 3 coherent state tomography is developed. In section 4, a resolution of the unity is obtained which provides a relation between Sudarshan's diagonal coherent state representation and Husimi-Kano $K$-function in the suggested tomographic setting. That identity resolution is then recovered in the context of the generalized phase space distributions associated with the Agarwal-Wolf $\Omega$-operator ordering [25, 26. In section 5, generic deformations of coherent states connected with $f$-oscillators, $q$-oscillators, and $s$-deformations associated to operator ordering are developed. In section 6 , photon number tomography is reviewed and the new scheme of deformed tomography with both nonlinearity and $s$-ordering is extended to photon number states. Some conclusions and perspectives are drawn in section 7. An Appendix presents a check of both the resolutions of the unity associated with the coherent state and the photon number tomographies and a derivation of the Mehler's formula in the holomorphic representation.

\section{Preliminaries}

In this section we give a short account of our previous works [12, 13, in which we have given an interpretation of quantum tomography in an abstract Hilbert space $\mathcal{H}$ by means of complete sets of rank-one projectors $\left\{P_{\mu}\right\}_{\mu \in M}$, where $M$ is a set of (multi-) parameters, discrete or continuous, collectively denoted by $\mu$. In general, a tomogram of a quantum state $|\psi\rangle$ is a positive real number $\mathcal{T}_{\psi}(\mu)$, depending on the parameter $\mu$ which labels a set of states $|\mu\rangle \in \mathcal{H}$, defined as

$$
\mathcal{T}_{\psi}(\mu):=|\langle\mu \mid \psi\rangle|^{2} .
$$

Our main idea was to regard the tomogram $\mathcal{T}_{\psi}(\mu)$ as a scalar product on the (Hilbert) space $\mathbb{H}$ of the rank-one projectors $|\mu\rangle\left\langle\mu\left|=P_{\mu} \rightarrow\right| P_{\mu}\right\rangle \in \mathbb{H}$ :

$$
\mathcal{T}_{\psi}(\mu)=\operatorname{Tr}\left(P_{\mu} \rho_{\psi}\right)=:\left\langle P_{\mu} \mid \rho_{\psi}\right\rangle .
$$

Equation (2) may readily be used to define the tomogram of any density operator $\hat{\rho}$ or any other (bounded) operator $\hat{A}$

$$
\mathcal{T}_{A}(\mu):=\operatorname{Tr}\left(P_{\mu} \hat{A}\right)=\left\langle P_{\mu} \mid A\right\rangle .
$$

Equation (33) shows in general that to any operator $\hat{A}$ a function $\langle\mu|\hat{A}| \mu\rangle$ of the variables $\mu$ corresponds in a given functional space. So, a tomograph may be thought of as a de-quantization, and in fact is an useful tool to study the 
quantum-classical transition by comparing classical limits of quantum tomograms with the corresponding classical tomograms [33. In the same sense, the inverse correspondence $\mathcal{T}_{A}(\mu) \rightarrow \hat{A}$ may be considered to give a quantization. The reconstruction of the operator $\hat{A}$ from its tomogram $\mathcal{T}_{A}(\mu)$ may be written as

$$
\hat{A}=\sum_{\mu \in M} \hat{G}_{\mu} \operatorname{Tr}\left(P_{\mu} \hat{A}\right) \Leftrightarrow|A\rangle=\sum_{\mu \in M}\left|G_{\mu}\right\rangle\left\langle P_{\mu} \mid A\right\rangle .
$$

In other words, the reconstruction of any operator is possible because the tomographic set $\left\{P_{\mu}\right\}_{\mu \in M}$ provides a resolution of the identity (super-) operator on $\mathbb{H}$ :

$$
\hat{\mathbb{I}}=\sum_{\mu \in M} \hat{G}_{\mu} \operatorname{Tr}\left(P_{\mu} \cdot\right)=\sum_{\mu \in M}\left|G_{\mu}\right\rangle\left\langle P_{\mu}\right| .
$$

We may then view $\left|G_{\mu}\right\rangle$ and $\left\langle P_{\mu}\right|$ as dual supervectors. For instance for the spin tomography, in the maximal qu-bit case $M$ is the Bloch sphere $S^{2}$ of all rank-one projectors and for any operator $\hat{A}$ holds [12]:

$$
\begin{aligned}
\hat{A} & =\int_{0}^{2 \pi} \int_{0}^{\pi} \hat{G}(\theta, \phi) \operatorname{Tr}(P(\theta, \phi) A) \sin \theta d \theta d \phi \\
& =\int_{0}^{2 \pi} \int_{0}^{\pi} P(\theta, \phi) \operatorname{Tr}(\hat{G}(\theta, \phi) A) \sin \theta d \theta d \phi,
\end{aligned}
$$

where, in matrix form,

$P(\theta, \phi)=\frac{1}{2}\left[\begin{array}{cc}1+\cos \theta & e^{-i \phi} \sin \theta \\ e^{i \phi} \sin \theta & 1-\cos \theta\end{array}\right] ; \hat{G}(\theta, \phi)=\frac{1}{4 \pi}\left[\begin{array}{cc}1+3 \cos \theta & 3 e^{-i \phi} \sin \theta \\ 3 e^{i \phi} \sin \theta & 1-3 \cos \theta\end{array}\right]$.

By definition, the set $\left\{P_{\mu}\right\}_{\mu \in M}$ is tomographic if it is complete in $\mathbb{H}$. A tomographic set determines a tomograph which is a functional, linear in the second argument

$$
\left(P_{\mu}, \hat{A}\right) \longrightarrow \mathcal{T}_{A}(\mu)=\operatorname{Tr}\left(P_{\mu} \hat{A}\right) .
$$

This definition is appropriate in the finite $n$-dimensional case, where

$$
|\mu\rangle \in \mathcal{H}_{n} \Leftrightarrow P_{\mu} \in \mathbb{H}_{n^{2}}=B\left(\mathcal{H}_{n}\right)=\mathcal{H}_{n} \otimes \mathcal{H}_{n},
$$

but in the infinite dimensional case the relation $\mathbb{H}=B(\mathcal{H})$ is no more valid and there are several relevant spaces, as the space of bounded operators $B(\mathcal{H})$ and that of compact operators $C(\mathcal{H})$, the space of Hilbert-Schmidt operators $\mathfrak{I}_{2}$ and that of trace-class operators $\mathfrak{I}_{1}$. Their mutual relations are:

$$
\mathfrak{I}_{1} \subset \mathfrak{I}_{2} \subset C(\mathcal{H}) \subset B(\mathcal{H}) .
$$

$B(\mathcal{H})($ and $C(\mathcal{H}))$ are Banach spaces, with the norm $\|A\|=\sup _{(\|\psi\|=1)}\|A \psi\|$, while $\mathfrak{I}_{2}$ is a Hilbert space with scalar product $\langle A \mid B\rangle=\operatorname{Tr}\left(A^{\dagger} B\right)$. Finally, $\mathfrak{I}_{1}$ is a Banach space with the norm $\|A\|_{1}=\operatorname{Tr}(|A|)$. The following inequalities hold true:

$$
\|A\| \leq\|A\|_{2} \leq\|A\|_{1} .
$$


So $\mathfrak{I}_{2}$, the only Hilbert space at our disposal to implement our definition of tomographic set, is endowed with a topology which, when restricted to the trace-class operators, is not equivalent to the topology of $\mathfrak{I}_{1}$. This may have serious consequences. In fact, in the finite dimensional case, the set $\left\{P_{\mu}\right\}_{\mu \in M}$ is complete iff

$$
\operatorname{Tr}\left(P_{\mu} A\right)=0 \quad \forall \mu \in M \Longrightarrow A=0 .
$$

Such a condition guarantees the full reconstruction of any observable from its tomograms. Now, in $\mathfrak{I}_{2}$, Eq. (9) reads:

$$
\left\langle P_{\mu} \mid A\right\rangle=0 \quad \forall \mu \in M \Longrightarrow A=0 \quad \& \quad A \in \mathfrak{I}_{2} .
$$

Then, as $\mathfrak{I}_{2}$ is a $*$-ideal in $B(\mathcal{H})$, there may exists a non-zero operator $B$, which is bounded but not Hilbert-Schmidt, such that

$$
\operatorname{Tr}\left(P_{\mu} B\right)=0 \quad \forall \mu \in M
$$

In other words, different observables may be tomographically separated only when their difference is Hilbert-Schmidt. Nevertheless there is a second case, when the set $\left\{P_{\mu}\right\}_{\mu \in M}$ of trace-class operators is complete even in $\mathfrak{I}_{1}$. Then, recalling that $\mathfrak{I}_{1}$ is a $*$-ideal in its dual space $B(\mathcal{H})$ :

$$
\mathfrak{I}_{1}^{*}=B(\mathcal{H}),
$$

the expression $\operatorname{Tr}\left(P_{\mu} A\right)$ is nothing but the value of the linear functional $\operatorname{Tr}(\cdot A)$ in $P_{\mu}$. Hence, Eq.(9) holds unconditionally

$$
\operatorname{Tr}\left(P_{\mu} A\right)=0 \quad \forall \mu \in M \Longrightarrow 0=\|\operatorname{Tr}(\cdot A)\|=\|A\| \Longrightarrow A=0 .
$$

Thus, the finest tomographies are those based on sets of rank-one projectors which are complete both in $\mathfrak{I}_{2}$ and in $\mathfrak{I}_{1}$. As a matter of fact, this is the case for the main tomographic sets, like the symplectic, the photon number and the coherent state tomographic sets.

From a geometrical point of view, tomographic sets are "skew" sets of projectors. In other words, we define a set of projectors to be skew when it spans the whole Hilbert space. Thus, any tomographic set is skew as it is complete. Besides, we define a set $\gamma$ of projectors to be locally skew in $P_{0}$, if it contains $P_{0}$ and any neighborhood of $P_{0}$ contains a skew subset of $\gamma$. Perhaps the simplest case of a tomographic set which is skew and locally skew is provided by the coherent state tomographic set discussed in the next sections.

\section{The coherent state tomography}

This tomographic set is generated by the displacement operators $\{\mathcal{D}(z)\}$ depending on a complex parameter $z$

$$
\mathcal{D}(z)=\exp \left(z \hat{a}^{\dagger}-z^{*} \hat{a}\right), \quad z \in \mathbb{C},
$$


which acting on the projector $|0\rangle\langle 0|$ of the vacuum Fock state, $\hat{a}|0\rangle=0$, yield the projectors

$$
|z\rangle\langle z|=\mathcal{D}(z)| 0\rangle\langle 0| \mathcal{D}(z)^{\dagger}, \quad z \in \mathbb{C},
$$

associated to the usual coherent states

$$
|z\rangle=\exp \left(-\frac{|z|^{2}}{2}\right) \exp \left(z \hat{a}^{\dagger}\right) \exp \left(-z^{*} \hat{a}\right)|0\rangle=\exp \left(-\frac{|z|^{2}}{2}\right) \sum_{j=0}^{\infty} \frac{z^{j}}{j !} \hat{a}^{\dagger j}|0\rangle .
$$

We recall that the coherent states are a (over-) complete set in the Hilbert space $\mathcal{H}$. Any bounded set containing a limit point $z_{0}$ in the complex $z$-plane defines a complete set of coherent states containing a limit point, the coherent state $\left|z_{0}\right\rangle$, in the Hilbert space $\mathcal{H}$. In particular, any Cauchy sequence $\left\{z_{k}\right\}$ of complex numbers defines a Cauchy sequence of coherent states $\left\{\left|z_{k}\right\rangle\right\}$, which is a complete set. The same holds for any extracted subsequence. This completeness property holds as $\exp \left(|z|^{2} / 2\right)\langle z \mid \psi\rangle$ is an entire analytic function of the complex variable $z^{*}$, for any $|\psi\rangle \in \mathcal{H}$. Then

$$
\left\langle z_{k} \mid \psi\right\rangle=0 \quad \forall k \Rightarrow|\psi\rangle=0
$$

because $z_{0}^{*}$ is a non-isolated zero of an analytic function.

Besides, any bounded operator $A$ may be completely reconstructed from its diagonal matrix elements $\left\langle z_{k}|A| z_{k}\right\rangle$. In fact, $\exp \left(|z|^{2} / 2+\left|z^{\prime}\right|^{2} / 2\right)\left\langle z|A| z^{\prime}\right\rangle$ is an analytical function of the complex variables $z^{*}, z^{\prime}$, so it is uniquely determined by its value $\exp \left(|z|^{2}\right)\langle z|A| z\rangle$ on the diagonal $z^{\prime}=z$. This is an entire function of the real variables $\Re z, \Im z$, which is in turn uniquely determined by its values on any set with an accumulation point.

The rank-one projectors associated to a complete set of coherent states are complete in the Hilbert space $\mathfrak{I}_{2}$. In particular, any Cauchy sequence $\left\{\left|z_{k}\right\rangle\right\}$ generates a tomographic set $\left\{\left|z_{k}\right\rangle\left\langle z_{k}\right|\right\}$. In fact, bearing in mind the previous remark on the reconstruction of a bounded operator, it results

$$
\operatorname{Tr}\left(A\left|z_{k}\right\rangle\left\langle z_{k}\right|\right)=\left\langle z_{k}|A| z_{k}\right\rangle=0 \quad \forall k \Rightarrow A=0 \quad \& \quad A \in B(\mathcal{H}) .
$$

This shows that a tomographic set of coherent state projectors is complete even in $\mathfrak{I}_{1}$. So it is skew. Moreover, any extracted subsequence $\left\{\left|z_{k_{n}}\right\rangle\left\langle z_{k_{n}}\right|\right\}$ is again complete, so $\left\{\left|z_{k}\right\rangle\left\langle z_{k}\right|\right\}$ is locally skew in its limit point. In the next section we address the case when $z$ varies in the whole complex plane.

\section{The resolution of unity generated by the co- herent state tomographic set}

It is possible to interpret the well known Husimi-Kano $K$-symbol of a (bounded) operator $\hat{A}$ as the coherent state (CS) tomogram of $\hat{A}$ :

$$
K_{A}(z):=\langle z|\hat{A}| z\rangle=: \operatorname{Tr}(|z\rangle\langle z| \hat{A}) .
$$


In particular, when $\hat{A}$ is chosen as a density operator $\hat{\rho}$, the identity holds

$$
\int \frac{d^{2} z}{\pi}\langle z|\hat{\rho}| z\rangle=\operatorname{Tr}(\hat{\rho})=1
$$

which allows for the probabilistic interpretation of the CS tomography. As a matter of fact [14 the $K$-symbol exists also for a number of non-bounded operators. The CS tomographic set is complete both in $\mathfrak{I}_{2}$, the space of HilbertSchmidt operators, and in $\mathfrak{I}_{1}$, the space of trace class operators acting on the space of states. In fact, the formulae

$$
\hat{A}=\int \frac{d^{2} z}{\pi} \frac{d^{2} z^{\prime}}{\pi}\left\langle z|\hat{A}| z^{\prime}\right\rangle|z\rangle\left\langle z^{\prime}\right|
$$

and $[25]$

$$
\left\langle z|\hat{A}| z^{\prime}\right\rangle=\mathrm{e}^{-\frac{|z|^{2}+\left|z^{\prime}\right|^{2}}{2}} \sum_{n, m=0}^{\infty} \frac{\left(z^{*}\right)^{n}\left(z^{\prime}\right)^{m}}{n ! m !}\left[\frac{\partial^{n+m}}{\partial z^{* n} \partial z^{m}}\left(\mathrm{e}^{|z|^{2}}\langle z|\hat{A}| z\rangle\right)\right]_{\substack{z^{*}=0 \\ z=0}}
$$

show that if the tomograms $\langle z|\hat{A}| z\rangle$ of a bounded operator $\hat{A}$ vanish for any $z \in \mathbb{C}$, then $\hat{A}$ is the zero operator. Equation (19) is implicit in Eq. (6) of Sudarshan Ref. [7.

So, a resolution of the unity exists, which allows for the full reconstruction of any (bounded) operator from its CS tomograms. We are interested in the explicit determination of such a formula. Now, the Sudarshan's diagonal coherent state representation $\phi_{A}(z)$ of an operator $\hat{A}$ is defined through the equation

$$
\hat{A}=\int \frac{d^{2} z}{\pi} \phi_{A}(z)|z\rangle\langle z| .
$$

Bearing in mind the previous qu-bit reconstruction formula, Eq.(7), we may note the analogy with Eq.(20) defining $\phi_{A}(z)$. Then, if our guess is right, we need to get explicitly the first form of the reconstruction formula, Eq.(6). In other words, we have to invert the well-known relation:

$$
K_{A}\left(z^{\prime}\right)=\left\langle z^{\prime}|\hat{A}| z^{\prime}\right\rangle=\int \frac{d^{2} z}{\pi} \phi_{A}(z)\left|\left\langle z \mid z^{\prime}\right\rangle\right|^{2}=\int \frac{d^{2} z}{\pi} \phi_{A}(z) \mathrm{e}^{-\left|z-z^{\prime}\right|^{2}}
$$

which follows at once from Eq.(20) defining $\phi_{A}(z)$. This relation shows that $K_{A}\left(z^{\prime}\right)$ is given by the convolution product of $\phi_{A}$ times a gaussian function. Then, denoting with $K_{A}\left(z_{R}^{\prime}, z_{I}^{\prime}\right)$ and $\phi_{A}\left(z_{R}, z_{I}\right)$ the $K$ and $\phi$ symbols, with $z^{\prime}=z_{R}^{\prime}+i z_{I}^{\prime} ; z=z_{R}+i z_{I}$, the Fourier transform [27] of Eq. (21) reads:

$$
\begin{aligned}
& \int \frac{d z_{R}^{\prime} d z_{I}^{\prime}}{2 \pi} K_{A}\left(z_{R}^{\prime}, z_{I}^{\prime}\right) \mathrm{e}^{-i\left(\xi z_{R}^{\prime}+\eta z_{I}^{\prime}\right)}= \\
& \int \frac{d z_{R}^{\prime} d z_{I}^{\prime}}{2 \pi} \int \frac{d z_{R} d z_{I}}{\pi} \phi_{A}\left(z_{R}, z_{I}\right) \mathrm{e}^{-\left(z_{R}-z_{R}^{\prime}\right)^{2}-\left(z_{I}-z_{I}^{\prime}\right)^{2}} \mathrm{e}^{-i\left(\xi z_{R}^{\prime}+\eta z_{I}^{\prime}\right)}
\end{aligned}
$$


and we readily obtain

$$
\tilde{K}_{A}(\xi, \eta)=\mathrm{e}^{-\left(\xi^{2}+\eta^{2}\right) / 4} \tilde{\phi}_{A}(\xi, \eta),
$$

from which

$$
\tilde{\phi}_{A}(\xi, \eta)=\mathrm{e}^{\left(\xi^{2}+\eta^{2}\right) / 4} \tilde{K}_{A}(\xi, \eta)
$$

that formally yields

$$
\phi_{A}\left(z_{R}, z_{I}\right)=\int \frac{d \xi d \eta}{2 \pi} \mathrm{e}^{\left(\xi^{2}+\eta^{2}\right) / 4} \tilde{K}_{A}(\xi, \eta) \mathrm{e}^{i\left(\xi z_{R}+\eta z_{I}\right)} .
$$

The presence of the anti-gaussian factor shows that the inverse Fourier transform of $\tilde{\phi}_{A}(\xi, \eta)$ exists only when the asymptotic decay of $\tilde{K}_{A}(\xi, \eta)$ is faster than the growth of $\mathrm{e}^{\left(\xi^{2}+\eta^{2}\right) / 4}$. However, the integral always exists as a distribution, as was proven in Ref.[15]. By virtue of this remark, we may go on and substitute the previous expression into Eq.(20) getting

$$
\begin{gathered}
\hat{A}=\int \frac{d^{2} z}{\pi}\left[\int \frac{d \xi d \eta}{2 \pi} \mathrm{e}^{\left(\xi^{2}+\eta^{2}\right) / 4} \tilde{K}_{A}(\xi, \eta) \mathrm{e}^{i\left(\xi z_{R}+\eta z_{I}\right)}\right]|z\rangle\langle z|= \\
\int \frac{d^{2} z}{\pi} \quad\left[\int \frac{d \xi d \eta}{2 \pi} \int \frac{d z_{R}^{\prime} d z_{I}^{\prime}}{2 \pi} K_{A}\left(z_{R}^{\prime}, z_{I}^{\prime}\right) \mathrm{e}^{\left(\xi^{2}+\eta^{2}\right) / 4} \mathrm{e}^{i\left[\xi\left(z_{R}-z_{R}^{\prime}\right)+\eta\left(z_{I}-z_{I}^{\prime}\right)\right]}\right]|z\rangle\langle z|
\end{gathered}
$$

Upon interchanging the order of integration, we may write the expected reconstruction formula as

$$
\hat{A}=\int \frac{d^{2} z^{\prime}}{\pi} \hat{G}\left(z^{\prime}\right) K_{A}\left(z^{\prime}\right)
$$

where the Gram-Schmidt operator $\hat{G}\left(z^{\prime}\right)$ reads:

$$
\hat{G}\left(z^{\prime}\right):=\int \frac{d^{2} z}{2 \pi} \int \frac{d \xi d \eta}{2 \pi} \mathrm{e}^{\left(\xi^{2}+\eta^{2}\right) / 4} \mathrm{e}^{i\left[\xi\left(z_{R}-z_{R}^{\prime}\right)+\eta\left(z_{I}-z_{I}^{\prime}\right)\right]}|z\rangle\langle z| .
$$

In other words, the resolution of the unity generated by the CS tomographic set is

$$
\hat{\mathbb{I}}=\int \frac{d^{2} z^{\prime}}{\pi} \hat{G}\left(z^{\prime}\right) \operatorname{Tr}\left(\left|z^{\prime}\right\rangle\left\langle z^{\prime}\right| \cdot\right) .
$$

We observe that

$$
\begin{aligned}
& K_{G\left(z^{\prime}\right)}(z)=\left\langle z\left|\hat{G}\left(z^{\prime}\right)\right| z\right\rangle=\int \frac{d \xi d \eta}{2 \pi} \mathrm{e}^{i\left[-\xi z_{R}^{\prime}-\eta z_{I}^{\prime}\right]} \mathrm{e}^{\left(\xi^{2}+\eta^{2}\right) / 4} \tilde{K}_{|z\rangle\langle z|}(\xi, \eta) \\
= & \int \frac{d \xi d \eta}{2 \pi} \mathrm{e}^{i\left[-\xi z_{R}^{\prime}-\eta z_{I}^{\prime}\right]} \tilde{\phi}_{|z\rangle\langle z|}(\xi, \eta)=\phi_{|z\rangle\langle z|}\left(z^{\prime}\right) .
\end{aligned}
$$

This, substituted in the reconstruction formula Eq.(27), gives also the reproducing kernel formula

$$
K_{G\left(z^{\prime \prime}\right)}(z)=\int \frac{d^{2} z^{\prime}}{\pi}\left\langle z\left|\hat{G}\left(z^{\prime}\right)\right| z\right\rangle K_{G\left(z^{\prime \prime}\right)}\left(z^{\prime}\right)=\int \frac{d^{2} z^{\prime}}{\pi} K_{G\left(z^{\prime}\right)}(z) K_{G\left(z^{\prime \prime}\right)}\left(z^{\prime}\right)
$$


or equivalently

$$
\phi_{|z\rangle\langle z|}\left(z^{\prime \prime}\right)=\int \frac{d^{2} z^{\prime}}{\pi} \phi_{|z\rangle\langle z|}\left(z^{\prime}\right) \phi_{\left|z^{\prime}\right\rangle\left\langle z^{\prime}\right|}\left(z^{\prime \prime}\right)
$$

Since

$$
|z\rangle\left\langle z\left|=\int \frac{d^{2} z^{\prime}}{\pi} \phi_{|z\rangle\langle z|}\left(z^{\prime}\right)\right| z^{\prime}\right\rangle\left\langle z^{\prime}\right| \Leftrightarrow \phi_{|z\rangle\langle z|}\left(z^{\prime}\right)=\pi \delta\left(z-z^{\prime}\right)
$$

we remark that Eq. (30) amounts to the orthonormality relations

$$
K_{G\left(z^{\prime}\right)}(z)=\operatorname{Tr}\left(|z\rangle\langle z| \hat{G}\left(z^{\prime}\right)\right)=\pi \delta\left(z-z^{\prime}\right)
$$

between dual sets of supervectors.

In view of Eq.(19), we can use the previous expression of $K_{G}$ to check the reconstruction formula, Eq.(27), in matrix form as:

$$
\langle z|\hat{A}| z\rangle=\int \frac{d^{2} z^{\prime}}{\pi} K_{G\left(z^{\prime}\right)}(z) K_{A}\left(z^{\prime}\right)=K_{A}(z) .
$$

A check of the reconstruction formula in the position representation is given in the Appendix.

In view of the generalization of our results in the next section, it is expedient to make preliminarily contact with the generalized phase space distributions associated with the Agarwal-Wolf $\Omega$-operator ordering [25, 26]. In fact, in the context of that operator ordering theory, the $K$ and $\phi$ symbols, appearing in our direct and dual reconstruction formulae, are related to the Wick (i.e., normal) and anti-Wick ordering respectively. As it is well known, it is possible to express any operator $A\left(\hat{a}, \hat{a}^{\dagger}\right)$ in terms of a function $F_{A}\left(z, z^{*}\right)$ of two complex variables $u=z, v=z^{*}$, by means of an operator $\hat{\Omega}$ which may be realized as integral operator

$$
A\left(\hat{a}, \hat{a}^{\dagger}\right)=\int \frac{d^{2} z}{\pi} F_{A}\left(z, z^{*}\right) \Delta^{(\Omega)}\left(z-\hat{a}, z^{*}-\hat{a}^{\dagger}\right)
$$

where the $\Omega-$ ordered delta function

$$
\Delta^{(\Omega)}\left(z-\hat{a}, z^{*}-\hat{a}^{\dagger}\right)=\int \frac{d^{2} w}{\pi} \Omega\left(w, w^{*}\right) \exp \left(w\left(z^{*}-\hat{a}^{\dagger}\right)-w^{*}(z-\hat{a})\right)
$$

is obtained by the displacement operator and an entire analytic function $\Omega\left(w, w^{*}\right)$ of two complex variables, which characterizes the particular rule of association between $\hat{A}$ and $F_{A}$. For instance, the Wick ordered delta function $\Delta^{(W)}$ is given by $\Omega\left(w, w^{*}\right)=\exp \left(w w^{*} / 2\right)$, while the anti-Wick ordered $\Delta^{(\bar{W})}$ is given by the reciprocal function $\exp \left(-w w^{*} / 2\right)$.

The function $F_{A}$ is associated to the operator $\hat{A}$ via the anti- $\Omega$ - ordered delta function:

$$
F_{A}\left(z, z^{*}\right)=\operatorname{Tr}\left[A\left(\hat{a}, \hat{a}^{\dagger}\right) \Delta^{(\bar{\Omega})}\left(z-\hat{a}, z^{*}-\hat{a}^{\dagger}\right)\right]
$$


It is apparent the similarity between Eq. (36) and our previous reconstruction formulae, but Agarwal-Wolf formulae, in general, do not contain rank-one projectors, which instead characterize our tomographic theory. However, it results that the anti-Wick ordered delta function is a rank-one projector:

$$
\Delta^{(\bar{W})}\left(z-\hat{a}, z^{*}-\hat{a}^{\dagger}\right)=|z\rangle\langle z|,
$$

so that the Wick ordered delta function $\Delta^{(W)}$ is nothing but our Gram-Schmidt operator $\hat{G}$ of Eq.(28), and Eq.(38) yields the Husimi-Kano function $K_{A}$ while Eq.(36) is just our direct reconstruction formula Eq.(27). When exchanging the role of the two orderings one obtains respectively the Sudarshan's diagonal representation $\phi_{A}$ and the dual form of our reconstruction formula, Eq.(20).

\section{The $(f, s)$-deformed CS tomographic set}

The usual coherent states, that we have recast in a tomographic setting in the previous sections, date back to Schrödinger. We have defined them by applying the unitary family of the displacement operators $\{\mathcal{D}(z)\}$ on the vacuum state $|0\rangle$, such that $\hat{a}|0\rangle=0$. Recently, a number of so-called generalized coherent states (GCS) have been introduced, in connection with oscillator algebras different from the standard one. In particular, an interesting family of such GCS, related with deformed oscillator algebras, is the set of nonlinear CS [20, 21, 28, which arises from a deformation ruled by a class $\{f(\hat{n})\}$ of functions of the number operator. This class is the set of real functions such that

$$
0<c<[f(n)] !<c^{\prime}<\infty, \quad n \in \mathbb{N} ;[f(n)] !:=f(n) f(n-1) \ldots f(1) .
$$

Following [28, that we briefly recall mainly to establish notation, we assume that these functions satisfy some further condition: they are continuous and moreover

$$
f(n)=1, \quad n=0,-1,-2, \ldots .
$$

Now, introducing the selfadjoint $f$-deformation operator

$$
\mathcal{E}_{f}:=f(\hat{n}) \prod_{m=1}^{\infty} f(\hat{n}-m)=:[f(\hat{n})] !
$$

we have

$$
\mathcal{E}_{f}^{-1} \hat{a} \mathcal{E}_{f}=\hat{a} f(\hat{n})=: \hat{A} ; \quad \mathcal{E}_{f}^{-1} \hat{a}^{\dagger} \mathcal{E}_{f}=\frac{1}{f(\hat{n})} \hat{a}^{\dagger}=: \hat{A}_{f}^{\dagger}
$$

Then, the $f$-deformed displacement operator is

$$
\mathcal{D}_{f}(z)=\mathcal{E}_{f}^{-1} \mathcal{D}(z) \mathcal{E}_{f}=\exp \left(z \hat{A}_{f}^{\dagger}-z^{*} \hat{A}\right) .
$$

Note that $\hat{A}_{f}^{\dagger}$ appears instead of $\hat{A}^{\dagger}=f(\hat{n}) \hat{a}^{\dagger}$ in the deformed displacement operator. This $\hat{A}_{f}^{\dagger}$ obeys the commutation rule $\left[\hat{A}, \hat{A}_{f}^{\dagger}\right]=\left[\hat{a}, \hat{a}^{\dagger}\right]=1$. 
Now, it is possible to compose the $f$-deformation with the $s$-deformation of operator ordering [29, 30], so defining the $(f, s)$-deformed displacement operators as:

$$
\mathcal{D}_{f, s}(z):=\exp \left(\frac{1}{2} s z z^{*}\right) \mathcal{D}_{f}(z)=\exp \left(\frac{1}{2} s z z^{*}\right) \exp \left(z \hat{A}_{f}^{\dagger}-z^{*} \hat{A}\right),
$$

where $z \in \mathbb{C},-1 \leq s \leq 1$.

The $(f, s)$-deformed displacement operators, acting on the vacuum, generate the $(f, s)$-deformed coherent states:

$$
\begin{aligned}
& |z ; f, s\rangle:=\mathrm{e}^{(1+s)|z|^{2} / 2} N_{z, f} \mathcal{D}_{f}(z)|0\rangle=\mathrm{e}^{(1+s)|z|^{2} / 2} N_{z, f} \mathcal{E}_{f}^{-1} \mathcal{D}(z) \mathcal{E}_{f}|0\rangle \\
& =\mathrm{e}^{(1+s)|z|^{2} / 2} N_{z, f} \mathcal{E}_{f}^{-1} \mathcal{D}(z) f(0)|0\rangle=\mathrm{e}^{(1+s)|z|^{2} / 2} N_{z, f} \mathcal{E}_{f}^{-1}|z\rangle
\end{aligned}
$$

where the normalizing factor is

$$
N_{z, f}=\left[\sum_{k=0}^{\infty} \frac{|z|^{2 k}}{k !([f(k)] !)^{2}}\right]^{-1 / 2} .
$$

Now, we are ready for the tomographic setting of such deformed states. We define the $(f, s)$-deformed $K$-function of an operator $\hat{B}$ as:

$$
\begin{aligned}
& K_{B}^{f, s}(z):=\langle z ; f, s|\hat{B}| z ; f, s\rangle= \\
& \left\langle z\left|\mathrm{e}^{(1+s)|z|^{2} / 2} N_{z, f} \mathcal{E}_{f}^{-1} \hat{B} \mathrm{e}^{(1+s)|z|^{2} / 2} N_{z, f} \mathcal{E}_{f}^{-1}\right| z\right\rangle=: \mathrm{e}^{(1+s)|z|^{2}} N_{z, f}^{2} K_{B(f)}(z)
\end{aligned}
$$

where

$$
\hat{B}(f):=\mathcal{E}_{f}^{-1} \hat{B} \mathcal{E}_{f}^{-1} .
$$

So, the $(f, s)$-deformed $K$-function of an operator $\hat{B}$ is the $K$-function of the $f$-deformed operator $\hat{B}(f)$ times a known $c$-number.

Thus, from the reconstruction formula of the non-deformed CS tomography:

$$
\hat{B}(f)=\int \frac{d^{2} z}{\pi} \hat{G}(z) K_{B(f)}(z),
$$

we get the reconstruction formula for the $(f, s)$-deformed CS tomography:

$$
\begin{aligned}
\hat{B} & =\int \frac{d^{2} z}{\pi} \mathcal{E}_{f} \hat{G}(z) \mathcal{E}_{f} K_{B(f)}(z)=\int \frac{d^{2} z}{\pi}\left[\mathcal{E}_{f} \hat{G}(z) \mathcal{E}_{f} \mathrm{e}^{-(1+s)|z|^{2}} N_{z, f}^{-2}\right] K_{B}^{f, s}(z) \\
& =: \int \frac{d^{2} z}{\pi} \hat{G}^{f, s}(z) K_{B}^{f, s}(z) .
\end{aligned}
$$

Remembering the expression of $\hat{G}$, Eq.(28), we have explicitly the $(f, s)$-deformed Gram-Schmidt operator $\hat{G}^{f, s}$ as:

$$
\begin{aligned}
& \hat{G}^{f, s}(z)=\frac{\mathrm{e}^{-(1+s)|z|^{2}}}{N_{z, f}^{2}} \int \frac{d^{2} z^{\prime}}{2 \pi} \int \frac{d \xi d \eta}{2 \pi} \mathrm{e}^{\left(\xi^{2}+\eta^{2}\right) / 4} \mathrm{e}^{i\left[\xi\left(z_{R}^{\prime}-z_{R}\right)+\eta\left(z_{I}^{\prime}-z_{I}\right)\right]} \mathcal{E}_{f}\left|z^{\prime}\right\rangle\left\langle z^{\prime}\right| \mathcal{E}_{f} \\
= & \frac{\mathrm{e}^{-2(1+s)|z|^{2}}}{N_{z, f}^{4}} \int \frac{d^{2} z^{\prime}}{2 \pi} \int \frac{d \xi d \eta}{2 \pi} \mathrm{e}^{\left(\xi^{2}+\eta^{2}\right) / 4} \mathrm{e}^{i\left[\xi\left(z_{R}^{\prime}-z_{R}\right)+\eta\left(z_{I}^{\prime}-z_{I}\right)\right]} \mathcal{E}_{f}^{2}\left|z^{\prime} ; f, s\right\rangle\left\langle z^{\prime} ; f, s\right| \mathcal{E}_{f}^{2}
\end{aligned}
$$


We recall [28] that $\mathcal{E}_{f}$ is selfadjoint and moreover $\mathcal{E}_{f} \mathcal{E}_{f}^{\dagger}=\mathcal{E}_{f}^{2}$ is a bounded positive operator (with bounded inverse) which satisfies the intertwining condition:

$$
\hat{A}^{\dagger} \mathcal{E}_{f}^{2}=\mathcal{E}_{f}^{2} \hat{A}_{f}^{\dagger} .
$$

In other words, the operator $\hat{A}_{f}^{\dagger}$ is nothing but the adjoint of the $f$-deformed operator $\hat{A}$ with respect to a new scalar product in our carrier Hilbert space $\mathcal{H}$ :

$$
(\varphi, \psi)_{f}:=\left(\varphi, \mathcal{E}_{f}^{2} \psi\right) \Rightarrow(\hat{A} \varphi, \psi)_{f}=\left(\varphi, \hat{A}^{\dagger} \mathcal{E}_{f}^{2} \psi\right)=\left(\varphi, \hat{A}_{f}^{\dagger} \psi\right)_{f}
$$

So, a better notation would be $\hat{A}_{f}^{\dagger}$ instead of $\hat{A}_{f}^{\dagger}$, however we shall use the latter one.

The presence, in the expression of the deformed Gram-Schmidt operator, of the deformed projector

$$
\mathcal{E}_{f}^{2}\left|z^{\prime} ; f, s\right\rangle\left\langle z^{\prime} ; f, s\right| \mathcal{E}_{f}^{2}
$$

is reminiscent of the new deformed scalar product, and allows the representation:

$$
\begin{aligned}
& \left\langle w ; f, s\left|\hat{G}^{f, s}(z)\right| w^{\prime} ; f, s\right\rangle=\frac{\mathrm{e}^{-2(1+s)|z|^{2}}}{N_{z, f}^{4}} \times \\
& \int \frac{d^{2} z^{\prime}}{2 \pi} \int \frac{d \xi d \eta}{2 \pi} \mathrm{e}^{\left(\xi^{2}+\eta^{2}\right) / 4} \mathrm{e}^{i\left[\xi\left(z_{R}^{\prime}-z_{R}\right)+\eta\left(z_{I}^{\prime}-z_{I}\right)\right]}\left\langle w ; f, s \mid z^{\prime} ; f, s\right\rangle_{f}\left\langle z^{\prime} ; f, s \mid w^{\prime} ; f, s\right\rangle_{f} .
\end{aligned}
$$

When $w^{\prime}=w$, we get

$$
\left[K_{|w ; f, s\rangle\langle w ; f, s|}^{f, s}\right]_{f}\left(z^{\prime}\right):=\left\langle w ; f, s \mid z^{\prime} ; f, s\right\rangle_{f}\left\langle z^{\prime} ; f, s \mid w ; f, s\right\rangle_{f}=K_{\mathcal{E}_{f}^{2}|w ; f, s\rangle\langle w ; f, s| \mathcal{E}_{f}^{2}}^{f, s}\left(z^{\prime}\right)
$$

and

$$
\begin{aligned}
& K_{G^{f, s}(z)}^{f, s}(w)=\left\langle w ; f, s\left|\hat{G}^{f, s}(z)\right| w ; f, s\right\rangle \\
& =\frac{\mathrm{e}^{-2(1+s)|z|^{2}}}{N_{z, f}^{4}} \quad \int \frac{d \xi d \eta}{2 \pi} \mathrm{e}^{i\left[-\xi z_{R}-\eta z_{I}\right]} \mathrm{e}^{\left(\xi^{2}+\eta^{2}\right) / 4} \tilde{K}_{\mathcal{E}_{f}^{2}|w ; f, s\rangle\langle w ; f, s| \mathcal{E}_{f}^{2}}^{f,}(\xi, \eta) \\
& =\frac{\mathrm{e}^{-2(1+s)|z|^{2}}}{N_{z, f}^{4}} \quad \phi_{\mathcal{E}_{f}^{2}|w ; f, s\rangle\langle w ; f, s| \mathcal{E}_{f}^{2}}^{f, s}(z),
\end{aligned}
$$

which may be compared with the analogous formula of the standard CS case, Eq.(30).

Let us consider now an important case of particular deformations, the $q-$ deformations. They are defined by considering the so called $q-$ oscillators [16, 17. For $q$ - oscillators, the analog of annihilation and creation operators $\hat{a}, \hat{a}^{\dagger}$ are the operators $\hat{a}_{q}$ and $\hat{a}_{q}^{\dagger}$ which obey the relation:

$$
\hat{a}_{q} \hat{a}_{q}^{\dagger}-q \hat{a}_{q}^{\dagger} \hat{a}_{q}=q^{\hat{n}}, \quad \hat{n}=\hat{a}^{\dagger} \hat{a},
$$


where $q$ is a $c$-number. When $q \rightarrow 1$, the relation of Eq.(58) becomes the standard boson commutation relation. One can introduce $q$-coherent states studying the eigenvalue problem

$$
\hat{a}_{q}|z ; q\rangle=z|z ; q\rangle
$$

The properties of $q$-oscillators and $q$-coherent states were intensively discussed in the literature. As it was shown, e.g., in Ref.[21], the $q$-deformations are a particular case of $f$-deformations, with a specific function $f_{q}$ coding the $q$-nonlinearity of the $q$-oscillator vibrations, which reads

$$
f_{q}(\hat{n}):=\sqrt{\frac{\sinh \lambda \hat{n}}{\lambda \hat{n}}}, \quad q:=\mathrm{e}^{\lambda} .
$$

One has:

$$
\hat{a}_{q}=\hat{a} f_{q}(\hat{n}) .
$$

The operator $\hat{a}_{q}$ of Eq.(61) and its adjoint $\hat{a}_{q}^{\dagger}$ satisfy the relation of Eq.(58). As a result, all the previous formulae for the deformed coherent states are valid even for $q$-deformations. In particular, this holds for the reconstruction formula Eq.(51) and the associated resolution of the unity, in which we have only to replace $f$ by $f_{q}$.

\section{Photon number tomographies}

\subsection{The photon number tomographic set}

The photon number tomography is generated by the irreducible family $\left\{\hat{a}^{\dagger} \hat{a},\{\mathcal{D}(z)\}\right\}$, where the displacement operators $\{\mathcal{D}(z)\}$ act on the number operator $\hat{a}^{\dagger} \hat{a}$ of the harmonic oscillator, yielding the family of selfadjoint operators $\hat{T}(z)$ :

$$
\hat{T}(z)=\mathcal{D}(z) \hat{a}^{\dagger} \hat{a} \mathcal{D}(z)^{\dagger}, \quad z \in \mathbb{C}
$$

which has the spectrum of $\hat{a}^{\dagger} \hat{a}$, eigenvectors $|n z\rangle=\mathcal{D}(z)|n\rangle$ and associated projectors $|n z\rangle\langle n z|$.

We recall that the photon number projectors' set, containing the complete set of the coherent state projectors, is a tomographic set complete both in $\mathfrak{I}_{2}$ and $\mathfrak{I}_{1}$. For the same reason, any Cauchy sequence $\left\{\left|n z_{k}\right\rangle\left\langle n z_{k}\right|\right\}$ is locally skew in its limit point 13 .

The whole set of photon number projectors generates the resolution of the unity:

$$
\hat{\mathbb{I}}=\sum_{n=0}^{\infty} \int \frac{d^{2} z}{\pi} \hat{G}_{\lambda}(n, z) \operatorname{Tr}(|n z\rangle\langle n z| \cdot) .
$$

The Gram-Schmidt operator $\hat{G}_{\lambda}$ is given by

$$
\hat{G}_{\lambda}(n, z)=\frac{4}{1-\lambda^{2}}\left(\frac{\lambda+1}{\lambda-1}\right)^{n} \mathcal{D}(z)\left(\frac{\lambda-1}{\lambda+1}\right)^{\hat{a}^{\dagger} \hat{a}} \mathcal{D}^{\dagger}(z)
$$


Here $\lambda$ is a real parameter, $-1<\lambda<1$, which labels the family of equivalent kernels $\hat{G}_{\lambda}(n, z)$.

The check of the matrix form of the resolution of the unity, Eq.(63), in the position representation is given in the Appendix.

\subsection{The $(f, s)$-deformed photon number tomographic set}

We can rephrase the section on the $(f, s)$-deformed coherent states, by letting the $(f, s)$-deformed displacement operators act on the eigenstates $|n\rangle$ of the harmonic oscillator, so generating the $(f, s)$-deformed photon number states:

$$
\begin{aligned}
|n z ; f, s\rangle & :=\mathrm{e}^{(1+s)|z|^{2} / 2} N_{z, f} \mathcal{D}_{f}(z)|n\rangle=\mathrm{e}^{(1+s)|z|^{2} / 2} N_{z, f} \mathcal{E}_{f}^{-1} \mathcal{D}(z) \mathcal{E}_{f}|n\rangle \\
= & \mathrm{e}^{(1+s)|z|^{2} / 2} N_{z, f} \mathcal{E}_{f}^{-1} \mathcal{D}(z)[f(n)] !|n\rangle=\mathrm{e}^{(1+s)|z|^{2} / 2} N_{z, f}[f(n)] ! \mathcal{E}_{f}^{-1}|n z\rangle
\end{aligned}
$$

where the normalizing factor $N_{z, f}$ is again given by Eq.(47).

Now, we define the $(f, s)$-deformed $K$-function of an operator $\hat{B}$ as:

$$
\begin{aligned}
K_{B}^{f, s}(n z) & :=\langle n z ; f, s|\hat{B}| n z ; f, s\rangle \\
& =\mathrm{e}^{(1+s)|z|^{2}} N_{z, f}^{2}\left[f^{2}(n)\right] !\left\langle n z\left|\mathcal{E}_{f}^{-1} \hat{B} \mathcal{E}_{f}^{-1}\right| n z\right\rangle \\
& =: \mathrm{e}^{(1+s)|z|^{2}} N_{z, f}^{2}\left[f^{2}(n)\right] ! K_{B(f)}(n z) .
\end{aligned}
$$

where

$$
\hat{B}(f):=\mathcal{E}_{f}^{-1} \hat{B} \mathcal{E}_{f}^{-1} .
$$

Again, the $(f, s)$-deformed $K$-function of an operator $\hat{B}$ is the $K$-function of the $f$-deformed operator $\hat{B}(f)$ times a known $c$-number. Then, the reconstruction formula for any bounded operator $\hat{B}$ in the $(f, s)$-deformed photon number tomography reads:

$$
\hat{B}=\sum_{n=0}^{\infty} \int \frac{d^{2} z}{\pi} \mathcal{E}_{f} \hat{G}_{\lambda}(n z) \mathcal{E}_{f} K_{B(f)}(n z)=: \sum_{n=0}^{\infty} \int \frac{d^{2} z}{\pi} \hat{G}_{\lambda}^{f, s}(n z) K_{B}^{f, s}(n z) .
$$

with

$$
\begin{aligned}
\hat{G}_{\lambda}^{f, s}(n z) & =\frac{\mathrm{e}^{-(1+s)|z|^{2}}}{\left[f^{2}(n)\right] ! N_{z, f}^{2}} \mathcal{E}_{f} \hat{G}_{\lambda}(n z) \mathcal{E}_{f} \\
& =\frac{\mathrm{e}^{-(1+s)|z|^{2}}}{\left[f^{2}(n)\right] ! N_{z, f}^{2}} \frac{4}{1-\lambda^{2}}\left(\frac{\lambda+1}{\lambda-1}\right)^{n} \mathcal{E}_{f}^{2} \mathcal{D}_{f}(z)\left(\frac{\lambda-1}{\lambda+1}\right)^{\hat{A}_{f}^{\dagger} \hat{A}} \mathcal{D}_{f}^{-1}(z),
\end{aligned}
$$

which is readily obtained by using

$$
\mathcal{E}_{f} \mathcal{D}(z) \hat{a}^{\dagger} \hat{a} \mathcal{D}^{\dagger}(z) \mathcal{E}_{f}=\mathcal{E}_{f}^{2} \mathcal{D}_{f}(z) \hat{a}^{\dagger} \hat{a} \mathcal{D}_{f}(-z)=\mathcal{E}_{f}^{2} \mathcal{D}_{f}(z) \hat{A}_{f}^{\dagger} \hat{A} \mathcal{D}_{f}^{-1}(z)
$$




\section{Conclusions}

To conclude, we recapitulate the new results of this work. We have constructed for infinite dimensional Hilbert spaces the tomographic sets based on coherent states and Fock states, respectively, and proved decomposition of the identity operator acting on $B(\mathcal{H})$, the space of bounded operators on the underlying Hilbert space $\mathcal{H}$, in terms of rank-one projectors of the tomographic set and associated Gram-Schmidt operators.

The properties of linear and non-linear $f$-oscillators were used to develop new schemes of the coherent state tomography. The $f$-deformed tomographic projectors were shown to yield a resolution of the (super-) unity simply by means of a linear non-canonical operator which may be used to deform the scalar product definition of $\mathcal{H}$. The particular case of the deformed oscillators, namely $q$-deformed oscillators, were studied as example of the coherent state tomography.

We addressed the general problem of mapping an abstract Hilbert space on the set of fair probability distributions (tomograms) describing quantum states in the framework of probability picture of quantum mechanics.

We hope to extend the obtained results to the case of multimode quantum systems and entangled states in future papers.

Acknowledgements V. I. Man'ko thanks for hospitality and support INFN, Sezione di Napoli and University "Federico II" of Naples.

E. C. G. Sudarshan's work was supported by U.S.Navy-Office of Naval Research, Grants No. N00014-04-1-0336 and No. N00014-03-1-0639.

\section{Appendix}

\subsection{Checking the resolution of the unity for the coherent state tomography}

In the position representation the reconstruction formula Eq. (27) reads:

$$
\begin{aligned}
\left\langle x|\hat{A}| x^{\prime}\right\rangle & =\int \frac{d^{2} z^{\prime}}{\pi}\left\langle x\left|\hat{G}\left(z^{\prime}\right)\right| x^{\prime}\right\rangle K_{A}\left(z^{\prime}\right) \\
& =\int d y d y^{\prime}\left[\int \frac{d^{2} z^{\prime}}{\pi}\left\langle x\left|\hat{G}\left(z^{\prime}\right)\right| x^{\prime}\right\rangle\left\langle z^{\prime} \mid y\right\rangle\left\langle y^{\prime} \mid z^{\prime}\right\rangle\right]\left\langle y|\hat{A}| y^{\prime}\right\rangle .
\end{aligned}
$$

So, we check the resolution of the unity generated by the CS tomographic set by showing that

$$
\int \frac{d^{2} z^{\prime}}{\pi}\left\langle x\left|\hat{G}\left(z^{\prime}\right)\right| x^{\prime}\right\rangle\left\langle y^{\prime} \mid z^{\prime}\right\rangle\left\langle z^{\prime} \mid y\right\rangle=\delta(x-y) \delta\left(x^{\prime}-y^{\prime}\right) .
$$

Recalling that

$$
\begin{array}{ll} 
& \left\langle y^{\prime} \mid z^{\prime}\right\rangle\left\langle z^{\prime} \mid y\right\rangle \\
=\frac{1}{\sqrt{\pi}} & \exp \left[i \sqrt{2} z_{I}^{\prime}\left(y^{\prime}-y\right)\right] \exp \left(-\frac{1}{2}\left[\left(y-\sqrt{2} z_{R}^{\prime}\right)^{2}+\left(y^{\prime}-\sqrt{2} z_{R}^{\prime}\right)^{2}\right]\right)(72)
\end{array}
$$


and

$$
\left\langle x\left|\hat{G}\left(z^{\prime}\right)\right| x^{\prime}\right\rangle=\int \frac{d^{2} z}{2 \pi} \int \frac{d \xi d \eta}{2 \pi} \mathrm{e}^{\left(\xi^{2}+\eta^{2}\right) / 4} \mathrm{e}^{i\left[\xi\left(z_{R}-z_{R}^{\prime}\right)+\eta\left(z_{I}-z_{I}^{\prime}\right)\right]}\langle x \mid z\rangle\left\langle z \mid x^{\prime}\right\rangle
$$

we may evaluate first the integral over $z_{I}$

$$
\int \frac{d z_{I}}{2 \pi} \exp \left[i z_{I}\left(\sqrt{2}\left(x-x^{\prime}\right)+\eta\right)\right]=\delta\left(\sqrt{2}\left(x-x^{\prime}\right)+\eta\right)
$$

and the integral over $z_{I}^{\prime}$

$$
\int \frac{d z_{I}^{\prime}}{2 \pi} \exp \left[i z_{I}^{\prime}\left(\sqrt{2}\left(y^{\prime}-y\right)-\eta\right)\right]=\delta\left(\sqrt{2}\left(y^{\prime}-y\right)-\eta\right)
$$

Then, apart from the pre-factor

$$
\frac{2}{\pi} \exp \left(-\frac{1}{2}\left(\left[y^{2}+y^{\prime 2}\right]+\left[x^{2}+x^{\prime 2}\right]\right)\right)
$$

we have to evaluate the integral

$$
\begin{aligned}
& \int d z_{R} d z_{R}^{\prime} \int \frac{d \xi d \eta}{2 \pi} \mathrm{e}^{\left(\xi^{2}+\eta^{2}\right) / 4} \mathrm{e}^{i\left[\xi\left(z_{R}-z_{R}^{\prime}\right)\right]} \delta\left(\sqrt{2}\left(x-x^{\prime}\right)+\eta\right) \delta\left(\sqrt{2}\left(y^{\prime}-y\right)-\eta\right) \times \\
& \mathrm{e}^{\left(-2 z_{R}^{\prime 2}+\sqrt{2} z_{R}^{\prime}\left(y+y^{\prime}\right)\right)} \mathrm{e}^{\left(-2 z_{R}^{2}+\sqrt{2} z_{R}\left(x+x^{\prime}\right)\right)} .
\end{aligned}
$$

The integral over $\eta$ yields

$$
\begin{aligned}
& \int d \eta \mathrm{e}^{\eta^{2} / 4} \delta\left(\sqrt{2}\left(x-x^{\prime}\right)+\eta\right) \delta\left(\sqrt{2}\left(y^{\prime}-y\right)-\eta\right) \\
= & \mathrm{e}^{\left(\frac{1}{4}\left[y^{\prime}-y\right]^{2}+\frac{1}{4}\left[x^{\prime}-x\right]^{2}\right)} \int d \eta \delta\left(\sqrt{2}\left(x-x^{\prime}\right)+\eta\right) \delta\left(\sqrt{2}\left(y^{\prime}-y\right)-\eta\right) \\
= & \exp \left(\frac{1}{4}\left[y^{\prime}-y\right]^{2}+\frac{1}{4}\left[x^{\prime}-x\right]^{2}\right) \frac{1}{\sqrt{2}} \delta\left(x-x^{\prime}+y^{\prime}-y\right),
\end{aligned}
$$

so that the pre-factor becomes

$$
\frac{2}{\pi} \exp \left(-\frac{1}{4}\left(\left[y^{\prime}+y\right]^{2}+\left[x^{\prime}+x\right]^{2}\right)\right) \frac{1}{\sqrt{2}} \delta\left(x-x^{\prime}+y^{\prime}-y\right),
$$

while the gaussian integrals over $z_{R}$ and $z_{R}^{\prime}$ give

$$
\begin{aligned}
& \int d z_{R} d z_{R}^{\prime} \mathrm{e}^{\left(-2 z_{R}^{\prime 2}+z_{R}^{\prime}\left[\sqrt{2}\left(y+y^{\prime}\right)-i \xi\right]\right)} \mathrm{e}^{\left(-2 z_{R}^{2}+z_{R}\left[\sqrt{2}\left(x+x^{\prime}\right)+i \xi\right]\right)} \\
= & \frac{\pi}{2} \mathrm{e}^{\left[\sqrt{2}\left(y+y^{\prime}\right)-i \xi\right]^{2} / 8} \mathrm{e}^{\left[\sqrt{2}\left(x+x^{\prime}\right)+i \xi\right]^{2} / 8} \\
= & \frac{\pi}{2} \exp \left(\frac{1}{4}\left(\left[y+y^{\prime}\right]^{2}+\left[x+x^{\prime}\right]^{2}\right)\right) \mathrm{e}^{-\xi^{2} / 4} \mathrm{e}^{i \xi\left(x+x^{\prime}-\left(y+y^{\prime}\right)\right) \sqrt{2} / 4} .
\end{aligned}
$$


Collecting all the terms, the pre-factor becomes

$$
\frac{1}{\sqrt{2}} \delta\left(x-y-\left(x^{\prime}-y^{\prime}\right)\right)
$$

and the last integral to evaluate is

$$
\int \frac{d \xi}{2 \pi} \mathrm{e}^{i \xi\left(x+x^{\prime}-\left(y+y^{\prime}\right)\right) \sqrt{2} / 4}=2 \sqrt{2} \delta\left(x+x^{\prime}-\left(y+y^{\prime}\right)\right) .
$$

So, we get the expected result

$$
2 \delta\left(x-y-\left(x^{\prime}-y^{\prime}\right)\right) \delta\left(x-y+\left(x^{\prime}-y^{\prime}\right)\right)=\delta(x-y) \delta\left(x^{\prime}-y^{\prime}\right) .
$$

\subsection{Checking the resolution of the unity for the photon number tomography}

Our aim is to check, for any allowed value of $\lambda$, the matrix form of the resolution

of unity for the photon number tomography, Eq. (63), which in the position representation reads

$$
\sum_{n=0}^{\infty} \int \frac{d^{2} z}{\pi}\left\langle y^{\prime} \mid n z\right\rangle\left\langle n z \mid x^{\prime}\right\rangle\left\langle x\left|\hat{G}_{\lambda}(n, z)\right| y\right\rangle=\delta\left(x-x^{\prime}\right) \delta\left(y-y^{\prime}\right) .
$$

With $z=(\nu+i \mu) / \sqrt{2}$, in the position representation $\langle y \mid n z\rangle$ is

$$
\begin{aligned}
\int d q\langle y|\mathcal{D}(z)| q\rangle\langle q \mid n\rangle & =\int d q \delta(y-q-\nu) \exp [i(\mu q+\mu \nu / 2)]\langle q \mid n\rangle \\
& =\exp [i(\mu y-\mu \nu / 2)]\langle y-\nu \mid n\rangle,
\end{aligned}
$$

where the $n$-th Hermite function $\langle q \mid n\rangle$ is

$$
\langle q \mid n\rangle=\left(\sqrt{\pi} 2^{n} n !\right)^{-1 / 2} \exp \left(-\frac{1}{2} q^{2}\right) H_{n}(q) .
$$

As we have shown in Ref.12, the matrix element of the Gram-Schmidt operator $\hat{G}_{\lambda}$ eventually reads:

$$
\begin{aligned}
\left\langle x\left|\hat{G}_{\lambda}(n, z)\right| y\right\rangle= & 4 \sin ^{2}\left(\frac{\tau}{2}\right) \frac{\mathrm{e}^{i \tau(n+1 / 2)}}{\sqrt{2 \pi i \sin \tau}} \exp [i \mu(y-x)] \times \\
\exp & \left(i\left[\left((x-\nu)^{2}+(y-\nu)^{2}\right) \frac{\cos \tau}{2 \sin \tau}-\frac{(x-\nu)(y-\nu)}{\sin \tau}\right]\right)
\end{aligned}
$$

where we have put

$$
\frac{\lambda+1}{\lambda-1}=\mathrm{e}^{i \tau},\left(\left.\tau\right|_{\lambda=0}=\pi\right) \Rightarrow \lambda=\frac{\mathrm{e}^{i \tau}+1}{\mathrm{e}^{i \tau}-1}=-i \cot \frac{\tau}{2} ; \frac{4}{1-\lambda^{2}}=4 \sin ^{2} \frac{\tau}{2} .
$$

Formulae (64), (87) correct the corresponding expressions given in Ref. [12]. 
Bearing in mind the expression of $\left\langle y^{\prime} \mid n z\right\rangle$ given by Eq.s (85]86) and recalling that $z=(\nu+i \mu) / \sqrt{2}$, so that $d^{2} z=d \mu d \nu / 2$, we have to evaluate

$$
\sum_{n=0}^{\infty} \int \frac{d \mu d \nu}{2 \pi} \exp \left[i \mu\left(y^{\prime}-x^{\prime}\right)\right]\left\langle y^{\prime}-\nu \mid n\right\rangle\left\langle n \mid x^{\prime}-\nu\right\rangle\left\langle x\left|G^{(\lambda)}(n, z)\right| y\right\rangle .
$$

Extracting the factor $\exp [i \mu(y-x)]$ from $\left\langle x\left|\hat{G}_{\lambda}(n, z)\right| y\right\rangle$, integration over $\mu$ yields

$$
\int \frac{d \mu}{2 \pi} \exp \left[i \mu\left(x^{\prime}-y^{\prime}\right)\right] \exp [i \mu(y-x)]=\delta\left(x^{\prime}-x-\left(y^{\prime}-y\right)\right) .
$$

It is possible to sum the series by means of the Mehler's formula, which is derived in the next subsection:

$$
\begin{aligned}
\sum_{n=0}^{\infty}\left(\frac{z}{2}\right)^{n} \frac{H_{n}(x) H_{n}(y)}{n !} & =\frac{1}{\sqrt{1-z^{2}}} \exp \left[\frac{z^{2}\left(x^{2}+y^{2}\right)-2 z x y}{z^{2}-1}\right], \\
(|z| & \leq 1, \quad z \neq \pm 1) .
\end{aligned}
$$

In our case $z=\mathrm{e}^{i \tau}$, so

$$
\frac{z}{z^{2}-1}=\frac{1}{z-z^{-1}}=\frac{-i}{2 \sin \tau} \quad ; \quad \frac{1}{\sqrt{1-z^{2}}}=\frac{z^{-1 / 2}}{\sqrt{z^{-1}-z}}=\frac{\mathrm{e}^{-i \tau / 2}}{\sqrt{-2 i \sin \tau}}
$$

and the Mehler's formula reads

$$
\frac{\mathrm{e}^{-i \tau / 2}}{\sqrt{-2 i \sin \tau}} \exp \left[\frac{-i}{2 \sin \tau}\left\{\mathrm{e}^{i \tau}\left[\left(x^{\prime}-\nu\right)^{2}+\left(y^{\prime}-\nu\right)^{2}\right]-2\left(x^{\prime}-\nu\right)\left(y^{\prime}-\nu\right)\right\}\right] \text {. }
$$

We are left with a prefactor

$$
4 \sin ^{2}\left(\frac{\tau}{2}\right) \frac{1}{\sqrt{\pi}} \frac{\mathrm{e}^{-i \tau / 2}}{\sqrt{-2 i \sin \tau}} \frac{\mathrm{e}^{i \tau / 2}}{\sqrt{2 \pi i \sin \tau}}=\frac{1}{\pi}\left|\tan \frac{\tau}{2}\right|
$$

and the integral

$$
\begin{aligned}
& \int d \nu \exp \left(-\frac{\sin \tau+i \mathrm{e}^{i \tau}}{2 \sin \tau}\left[\left(x^{\prime}-\nu\right)^{2}+\left(y^{\prime}-\nu\right)^{2}\right]+i \frac{\left(x^{\prime}-\nu\right)\left(y^{\prime}-\nu\right)}{\sin \tau}\right) \times \\
& \exp \left(i \frac{\cos \tau}{2 \sin \tau}\left[(x-\nu)^{2}+(y-\nu)^{2}\right]-i \frac{(x-\nu)(y-\nu)}{\sin \tau}\right) .
\end{aligned}
$$

The coefficient of $\nu^{2}$ vanishes:

$$
-\frac{\sin \tau+i \mathrm{e}^{i \tau}}{\sin \tau}+i \frac{\cos \tau}{\sin \tau}=0
$$

Then, apart from the prefactor

$$
\frac{\left|\tan \frac{\tau}{2}\right|}{\pi} \exp \left(-\frac{i \cos \tau}{2 \sin \tau}\left[x^{\prime 2}+y^{\prime 2}\right]+\frac{i \cos \tau}{2 \sin \tau}\left[x^{2}+y^{2}\right]+\frac{i\left(x^{\prime} y^{\prime}-x y\right)}{\sin \tau}\right),
$$


we have

$$
\begin{aligned}
& \int d \nu \exp \left(\nu\left[\frac{i \cos \tau}{\sin \tau}\left(x^{\prime}+y^{\prime}\right)-\frac{i \cos \tau}{\sin \tau}(x+y)-\frac{i\left(x^{\prime}+y^{\prime}-(x+y)\right)}{\sin \tau}\right]\right) \\
= & \int d \nu \exp \left[-i \nu\left(x^{\prime}+y^{\prime}-(x+y)\right) \tan \frac{\tau}{2}\right]=\frac{2 \pi}{\left|\tan \frac{\tau}{2}\right|} \delta\left(x^{\prime}+y^{\prime}-(x+y)\right) .
\end{aligned}
$$

Hence we get the expected product of delta functions

$$
2 \delta\left(x^{\prime}-x-\left(y^{\prime}-y\right)\right) \delta\left(x^{\prime}+y^{\prime}-(x+y)\right)=\delta\left(x^{\prime}-x\right) \delta\left(y^{\prime}-y\right) .
$$

In view of this result, we may assume $x^{\prime}=x, y^{\prime}=y$ in the prefactor

$$
\exp \left(-\frac{i \cos \tau}{2 \sin \tau}\left[x^{\prime 2}+y^{\prime 2}\right]+\frac{i \cos \tau}{2 \sin \tau}\left[x^{2}+y^{2}\right]+\frac{i\left(x^{\prime} y^{\prime}-x y\right)}{\sin \tau}\right) \rightarrow 1,
$$

so that we obtain just the resolution of unity of Eq.84).

\subsection{A deduction of the Mehler's formula in the holomor- phic representation}

Here we give a deduction of the Mehler's formula (1866) by using the holomorphic, i.e. Bargmann-Fock, representation of the harmonic oscillator wave functions:

$$
\begin{aligned}
\left\langle z \mid \psi_{n}\right\rangle & =\psi_{n}\left(z^{*}\right)=\frac{z^{* n}}{\sqrt{n !}}, \quad z=z_{R}+i z_{I} ; \\
\left\langle\psi_{m} \mid \psi_{n}\right\rangle & =\int \frac{z^{m}}{\sqrt{m !}} \frac{z^{* n}}{\sqrt{n !}} d \mu_{a}=\delta_{n m}, \quad d \mu_{a}:=\mathrm{e}^{-z^{*} z} \frac{d z_{R} d z_{I}}{\pi}=\mathrm{e}^{-z^{*} z} \frac{d z^{*} d z}{2 \pi i} .
\end{aligned}
$$

Representation of Dirac's delta function reads:

$$
f\left(z^{*}\right)=\int \mathrm{e}^{z^{*} z^{\prime}} f\left(z^{*}\right) d \mu_{z^{\prime}} .
$$

We use this representation of the delta function for evaluating the integral

$$
\begin{aligned}
J(x, y ; \zeta) & =\int \mathrm{e}^{\sqrt{2} x z-z^{2} / 2} \mathrm{e}^{\zeta z^{*} z^{\prime}} \mathrm{e}^{\sqrt{2} y z^{\prime *}-z^{\prime * 2} / 2} d \mu_{z} d \mu_{z^{\prime}} \\
& =\int \mathrm{e}^{\sqrt{2} x z-z^{2} / 2} \mathrm{e}^{\sqrt{2} \zeta y z^{*}-\zeta^{2} z^{* 2} / 2} d \mu_{z} .
\end{aligned}
$$

Then we get for $J(x, y ; \zeta)$ the gaussian integral:

$$
\begin{aligned}
& \int \exp \left[-\frac{1}{2}\left(z, z^{*}\right)\left[\begin{array}{cc}
1 & 1 \\
1 & \zeta^{2}
\end{array}\right]\left(\begin{array}{c}
z \\
z^{*}
\end{array}\right)+\sqrt{2}(x, \zeta y)\left(\begin{array}{c}
z \\
z^{*}
\end{array}\right)\right] \frac{d z^{*} d z}{2 \pi i} \\
= & {\left[-\operatorname{det}\left[\begin{array}{cc}
1 & 1 \\
1 & \zeta^{2}
\end{array}\right]\right]^{-\frac{1}{2}} \exp \left[(x, \zeta y)\left[\begin{array}{cc}
1 & 1 \\
1 & \zeta^{2}
\end{array}\right]^{-1}\left(\begin{array}{c}
x \\
\zeta y
\end{array}\right)\right] . }
\end{aligned}
$$


Eventually

$$
J(x, y ; \zeta)=\frac{1}{\sqrt{1-\zeta^{2}}} \exp \left[\frac{\zeta^{2}\left(x^{2}+y^{2}\right)-2 \zeta x y}{\zeta^{2}-1}\right] .
$$

On the other side, remembering that the generating function of the Hermite polynomials in the position representation is

$$
\mathrm{e}^{\sqrt{2} x z-z^{2} / 2}=\sum_{n=0}^{\infty} \frac{H_{n}(x)}{n !}\left(\frac{z}{\sqrt{2}}\right)^{n},
$$

we have that Eq. (100) also reads:

$$
J(x, y ; \zeta)=\sum_{n, m=0}^{\infty} \frac{H_{n}(x) H_{m}(y)}{2^{(n+m) / 2} n ! m !} \int z^{n} \mathrm{e}^{\zeta z^{*} z^{\prime}}\left(z^{*}\right)^{m} d \mu_{z} d \mu_{z^{\prime}} .
$$

Again we use the Dirac's delta and get for $J(x, y ; \zeta)$ :

$$
\sum_{n, m=0}^{\infty} \frac{H_{n}(x) H_{m}(y)}{2^{(n+m) / 2} n ! m !} \int z^{n}\left(\zeta z^{*}\right)^{m} d \mu_{z}=\sum_{n, m=0}^{\infty} \frac{\zeta^{m} H_{n}(x) H_{m}(y)}{2^{(n+m) / 2} \sqrt{n ! m !}}\left\langle\psi_{n} \mid \psi_{m}\right\rangle
$$

By comparing this expression of $J(x, y ; \zeta)$ with the previous one of Eq. (102) we finally obtain the Mehler's formula

$$
\sum_{n=0}^{\infty}\left(\frac{\zeta}{2}\right)^{n} \frac{H_{n}(x) H_{n}(y)}{n !}=\frac{1}{\sqrt{1-\zeta^{2}}} \exp \left[\frac{\zeta^{2}\left(x^{2}+y^{2}\right)-2 \zeta x y}{\zeta^{2}-1}\right] .
$$

The radius of convergence of the series is 1 , but from the previous evaluation we get that the values $|\zeta|=1, \zeta \neq \pm 1$ are allowed because

$$
\operatorname{det}\left[\begin{array}{cc}
1 & 1 \\
1 & \zeta^{2}
\end{array}\right] \neq 0
$$

The limits $\zeta \rightarrow \pm 1$ are

$$
\lim _{\zeta \rightarrow \pm 1} \frac{1}{\sqrt{1-\zeta^{2}}} \exp \left[\frac{\zeta^{2}\left(x^{2}+y^{2}\right)-2 \zeta x y}{\zeta^{2}-1}\right]=\sqrt{\pi} \delta(x \mp y) .
$$

\section{References}

[1] D. F. Styer, M. S. Balkin, K. M. Becker, M. R. Burns, C. E. Dudley, S. T. Forth, J. S. Gaumer, M. A. Kramer, D. C. Oertel, L. H. Park, M. T. Rinkoski, C. T. Smith, T. D. Wotherspoon, Am. J. Phys. 70, 288 (2002).

[2] L.D. Landau, Z. Phys. 45, 430 (1927). 
[3] J. von Neumann, Nach. Ges. Wiss. Göttingen 11, 245 (1927).

[4] E. Wigner, Phys. Rev. 40, 749 (1932).

[5] K. Husimi, Proc. Phys. Math. Soc. Jpn. 22, 264 (1940).

[6] Y. Kano, J. Math. Phys. 6, 1913 (1965) .

[7] E. C. G. Sudarshan, Phys. Rev. Lett. 10, 277 (1963).

[8] R. J. Glauber, Phys. Rev. Lett. 10, 84 (1963) ; Phys. Rev. 131, 2766 (1963).

[9] J. Bertrand and P. Bertrand, Found. Phys. 17, 397 (1987).

[10] K. Vogel and H. Risken, Phys. Rev. A 40, 2847 (1989).

[11] S. Mancini, V. I. Man'ko and P. Tombesi, Quantum Semiclass. Opt. 7, 615 (1995).

[12] V. I. Man'ko, G. Marmo, A. Simoni, A. Stern, E. C. G. Sudarshan, F. Ventriglia, Phys. Lett. A 351, 1 (2006).

[13] V. I. Man'ko, G. Marmo, A. Simoni, F. Ventriglia, Open Systems \& Information Dynamics, 13, 239 (2006).

[14] J. R. Klauder and E. C. G. Sudarshan, Fundamentals of Quantum Optics, (Benjamin, New York, 1968).

[15] C. L. Mehta and E. C. G. Sudarshan, Phys. Rev. 138, B274 (1965).

[16] L. Biedenharn, J. Phys. A: Math. Gen.22, L873 (1989).

[17] A. Mac Farlane, J. Phys. A: Math. Gen. 22, 4581 (1989).

[18] V. I. Man'ko, G. Marmo, E. C. G. Sudarshan, F. Zaccaria, Phys. Scr. 55, 528 (1997).

[19] V. I. Man'ko, G. Marmo, S. Solimeno, F. Zaccaria, Int. J. Mod. Phys. A 8, 3577 (1993).

[20] R. L. de Matos Filho and W. Vogel, Phys. Rev. A 54, 4560 (1996).

[21] V. I. Man'ko, G. Marmo, E. C. G. Sudarshan, F. Zaccaria, p. 421 in fOscillators, Proc. IV Wigner Symp., Guadalahara, Mexico, July 1995, Ed.s N. M. Atakishiyev, T. M. Seligman, K. B. Wolf, (World Scientific, Singapore, 1996).

[22] K. Banaszek and K. Wódkiewicz, Phys, Rev. Lett. 76, 4344 (1996).

[23] S. Wallentowitz and W. Vogel, Phys. Rev. A 53, 4528 (1996).

[24] S. Mancini, P. Tombesi and V. I. Man'ko, Europhys. Lett. 37, 79 (1997). 
[25] L. Mandel and E. Wolf, Optical coherence and Quantum Optics, (Cambridge University Presss, Cambridge, 1995).

[26] G. S. Agarwal and E. Wolf, Phys, Rev. Lett. 21, 180 (1968); Phys, Rev. D 2, 2161 (1970); Phys, Rev. D 2, 2187 (1970); Phys, Rev. D 2, 2206 (1970).

[27] W. P. Schleich, Quantum Optics in Phase Space, (Wiley-VCH, Berlin, 2001).

[28] P. Aniello, V. I. Man'ko, G. Marmo, S. Solimeno anf F. Zaccaria, J. Opt. B: Quantum Semiclass. Opt. 2, 718 (2000).

[29] E. C. G. Sudarshan, Structure of Dynamical Theories, in Proc. Brandeis Summer Institute on Theoretical Physics (1961); notes compiled by G. Carmi; (W. A. Benjamin Inc., New York, 1962).

[30] K. E. Cahill and R. J. Glauber, Phys. Rev. 176, 1857 (1968); Phys. Rev. 177, 1882 (1969).

[31] S. Mancini, V. I. Man'ko and P. Tombesi, Phys. Lett. A 213, 1 (1996); Found. Phys. 27, 81 (1997).

[32] G. M. D'Ariano, S. Mancini, V. I. Man'ko and P. Tombesi, Quantum Semiclass. Opt. 8, 1017 (1996).

[33] V. I. Man'ko, G. Marmo, A. Simoni, A. Stern, F. Ventriglia, Phys. Lett. A 343, 251 (2005). 